\title{
Willingness to pay for COVID-19 vaccination in China
}

Most adults in China are willing to pay for COVID-19 vaccination, but most also believe that the government and health insurance should pay for all or some of the cost of vaccination, according to findings of a study published in Vaccine.

An online survey was conducted in adults in China in March 2020 to investigate willingness-to-pay (WTP) and financing preferences for COVID-19 vaccination during the pandemic; 2058 respondents completed the survey.

The 2019 family income ranged from $\$ 7246$ to $\$ 21739$ in 51\% of respondents, $62 \%$ worked in workplaces with 30 or more employees, and 70\% believed that the COVID-19 pandemic was declining in China.

The mean and median WTP for full COVID-19 vaccination was $\$ 36.8^{*}$ and $\$ 14.5$, respectively.

The government and health insurance should pay for all of some of the costs of COVID-19 vaccination, reported $90.9 \%$ and $78.0 \%$ of respondents, respectively. However, $84.3 \%$ of respondents said that individuals should pay some $(77.9 \%)$ or all $(6.4 \%)$ of the vaccination costs.

Annual family income, employee size, and whether the respondent believed the pandemic is declining, influenced the WTP of respondents.

"The public stated individuals, governments and insurances could all be considered in financing COVID-19 vaccination. But to suggest an effective and optimal financing strategy in controlling and preventing the COVID-19 pandemic, the public health perspective with equal access to COVID-19 vaccination should be prioritized to ensure a high vaccination rate, with further and comprehensive information needed," concluded the investigators.

* 2020 US dollars

Wang J, et al. Willingness to pay and financing preferences for COVID-19 vaccination in China. Vaccine : 27 Feb 2021. Available from: URL: http://doi.org/10.1016/ j.vaccine.2021.02.060 